\title{
Influence of Geant4 parameters on proton dose distribution
}

\author{
Asad Merouani' ${ }^{1}$, Naima El-Khayati ${ }^{1}$, Gabriel Amoros ${ }^{2}$ \\ ${ }^{I}$ Department of Physics, Faculty of Science, Mohammed V University, Rabat, Morocco \\ ${ }^{2}$ Instituto de Física Corpuscular, Consejo Superior de Investigaciones Cientificas (CSIC/UV), Valencia, Spain
}

[Presented at the $4^{\text {th }}$ African Regional IRPA congress (AFRIRPA04), which was held from September 13-17, 2014 in Rabat, Morocco. This paper was reviewed and accepted by the scientific committee of the $4^{\text {th }}$ African Regional IRPA congress]

\section{Conference Proceeding}

\begin{abstract}
Purpose: The proton therapy presents a great precision during the radiation dose delivery. It is useful when the tumor is located in a sensitive area like brain or eyes. The Monte Carlo (MC) simulations are usually used in treatment planning system (TPS) to estimate the radiation dose. In this paper we are interested in estimating the proton dose statistical uncertainty generated by the MC simulations. Methods: Geant4 was used in the simulation of the eye's treatment room for $62 \mathrm{MeV}$ protons therapy, installed in the Istituto Nazionale Fisica Nucleare Laboratori Nazionali del Sud (LNS-INFN) facility in Catania. This code is a Monte Carlo based on software dedicated to simulate the passage of particles through the matter. In this work, we are interested in optimizing the Geant 4 parameters on energy deposit distribution by proton to achieve the spatial resolution of dose distribution required for cancer therapy. We propose various simulations and compare the corresponding dose distribution inside water to evaluate the statistical uncertainties. Results: The simulated Bragg peak, based on facility model is in agreement with the experimental data, The calculations show that the mean statistical uncertainty is less than $1 \%$ for a simulation set with $5 \times 10^{4}$ events, $10^{-3}$ mm production threshold and a $10^{-2} \mathrm{~mm}$ step limit. Conclusion: The set of Geant 4 cut and step limit values can be chosen in combination with the number of events to reach precision recommended from International Commission on Radiation Units and measurements (ICRU) in Monte Carlo codes for proton therapy treatment.
\end{abstract}

Keywords: Proton Therapy; Monte Carlo Simulation; Geant4: Dose Distribution; Statistical Uncertainties

\section{Introduction}

Geant 4 is a toolkit, developed to simulate the passage of particles through the matter based on Monte Carlo method. ${ }^{1}$ It is used for simulation of many physics applications in different areas, as well as the high energy nuclear experiment, accelerator physics, medical and space science studies. This code was used for medical physics simulations in different application such as hadron therapy, radiotherapy, and positron emission tomography (PET) \& single-photon emission computed tomography (SPECT) in Gate collaboration. Geant 4 is adapted to simulate the hadron therapy application due the physical processes that include electromagnetic and hadronic interactions.

The passage of charged particles through matter is characterized by the energy lost, which is derived into elastic and inelastic collision with atomic electrons of the material. This effect is responsible for the energy loss of heavy particles in matter. Lost energy is causing ionization or excitation of atom. ${ }^{2}$ In Geant 4 the calculation of the energy loss is done by using the Bethe-Bloch formula. The calculation of the continuous and discrete energy loss in a material is needed. The value of the kinetic energy cut-off or production threshold
(Ecut) below the energy loss is continuous and above it the energy loss is simulated by the production of secondary particle. ${ }^{3}$ The specific of Geant 4 is that threshold energy is set as a range in millimeters, to annul the dependence on material and particle type. Each range is calculated depending on the material for a defined G4Region or for the complete geometry. For electrons and positrons the conversion from range to kinetic energy is done according to the continuous slow down approximation (CSDA).

In Geant4, a limit on the step-size is imposed because of the continuous energy loss dependence of the cross sections. In Monte Carlo programs, the cross sections are approximately constant along step. The set of the step size should be small enough, to consider the cross section approximately constant along the step. In fact this set of steps requires an accurate simulation, but the computing time increases as the step-size decreases. In order to insure accurate simulation, the step sizes and production thresholds must be small. ${ }^{4}$ The best choice is to combine both of parameters, for allowing accurate simulation and reasonable computation time. 
In this work, we study the influence of Geant 4 parameters on energy deposit distribution by proton to achieve the spatial resolution of dose distribution ${ }^{5}$ required for cancer therapy. We propose various simulations to help users fix parameter values and understand their influence on accuracy of simulation and time of simulation.

\section{Methods and Materials}

In this paper, the model and the proton therapy simulations were performed with Geant4 Monte Carlo computing code. The simulation represents the eye's treatment room for proton therapy, installed at the LNS-INFN facility in Catania ${ }^{3}$, with $62 \mathrm{MeV}$ protons Figure 1. The passive method was used in the beam irradiation system to achieve a three dimensional dose distribution using a lateral beam-spreading ${ }^{6-7}$ system and a range modulating system. The model is one of Geant 4 advanced examples; simulate a box of water of $40 \times 40 \times$ $40 \mathrm{~mm}^{3}$ using Geant 4 version 10.0 Figure 2. The phantom was dividedinto a voxel size of $40 \times 40 \times 0.1 \mathrm{~mm}^{3}$ attached to the water box. The proton beam is a $62 \mathrm{MeV}$ monoenergetic beam with Gaussian shape. The physic list used is QGSP_BIC_HP with the Low Energy model package that allows electron and photon simulations down to $250 \mathrm{eV} .^{3}$ This physic list package is used for medical applications, and mainly for the studies using very low $\left(10^{-3} \mathrm{~mm}\right)$ production threshold. All the information and description of the physical models are given in the Physics Reference Manual. ${ }^{3}$ The lowest permitted parameters, ${ }^{4}$ production threshold of $10^{-3} \mathrm{~mm}$ and a step limit of $10^{-2} \mathrm{~mm}$, were chosen to achieve the most accurate results. The Figure 3 shows a comparison between Bragg peak simulation, with $10^{5}$ events, and measured data.

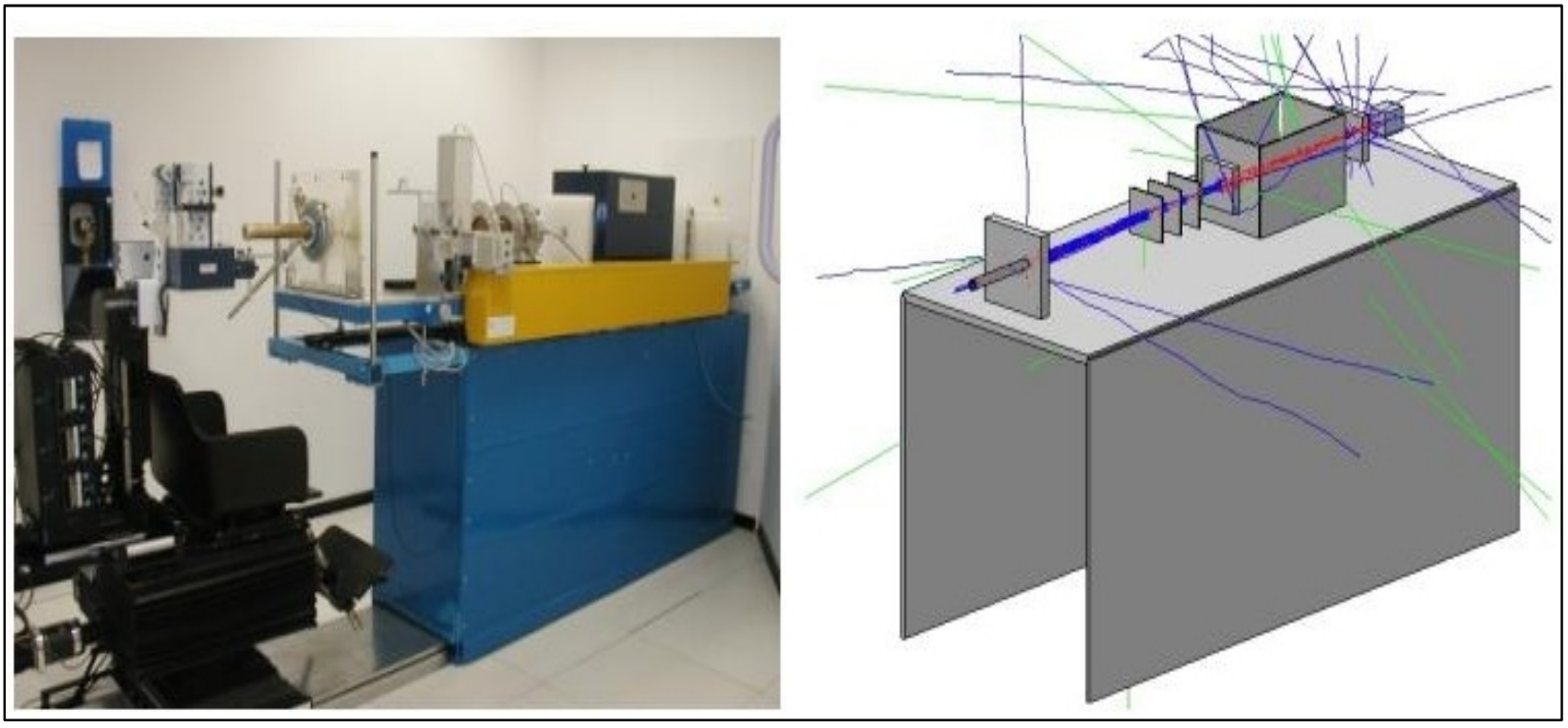

FIG. 1: The real proton therapy beam line installed at Laboratori Nazionali del Sud (INFN) in Catania.

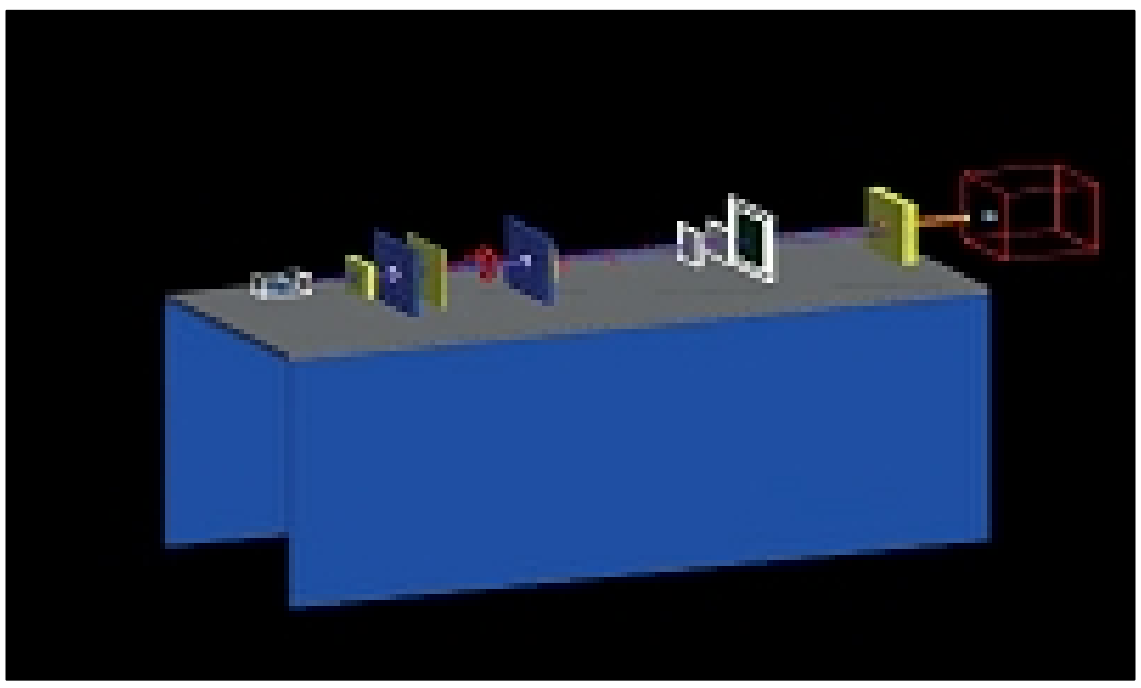

FIG. 2: Simulated model. 


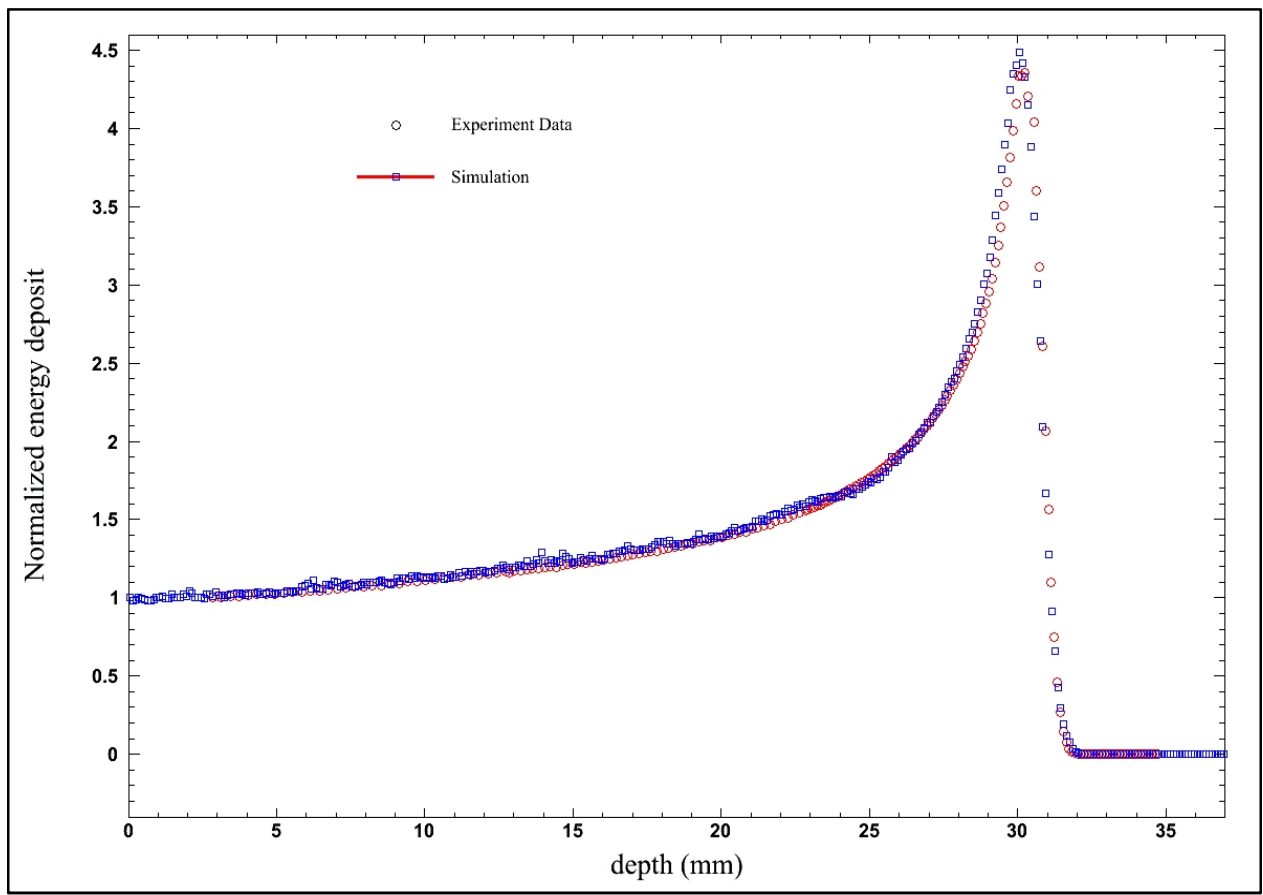

FIG. 3: Bragg peaks comparative plots: experimental data and calculation using the LNS-INFN facility model.

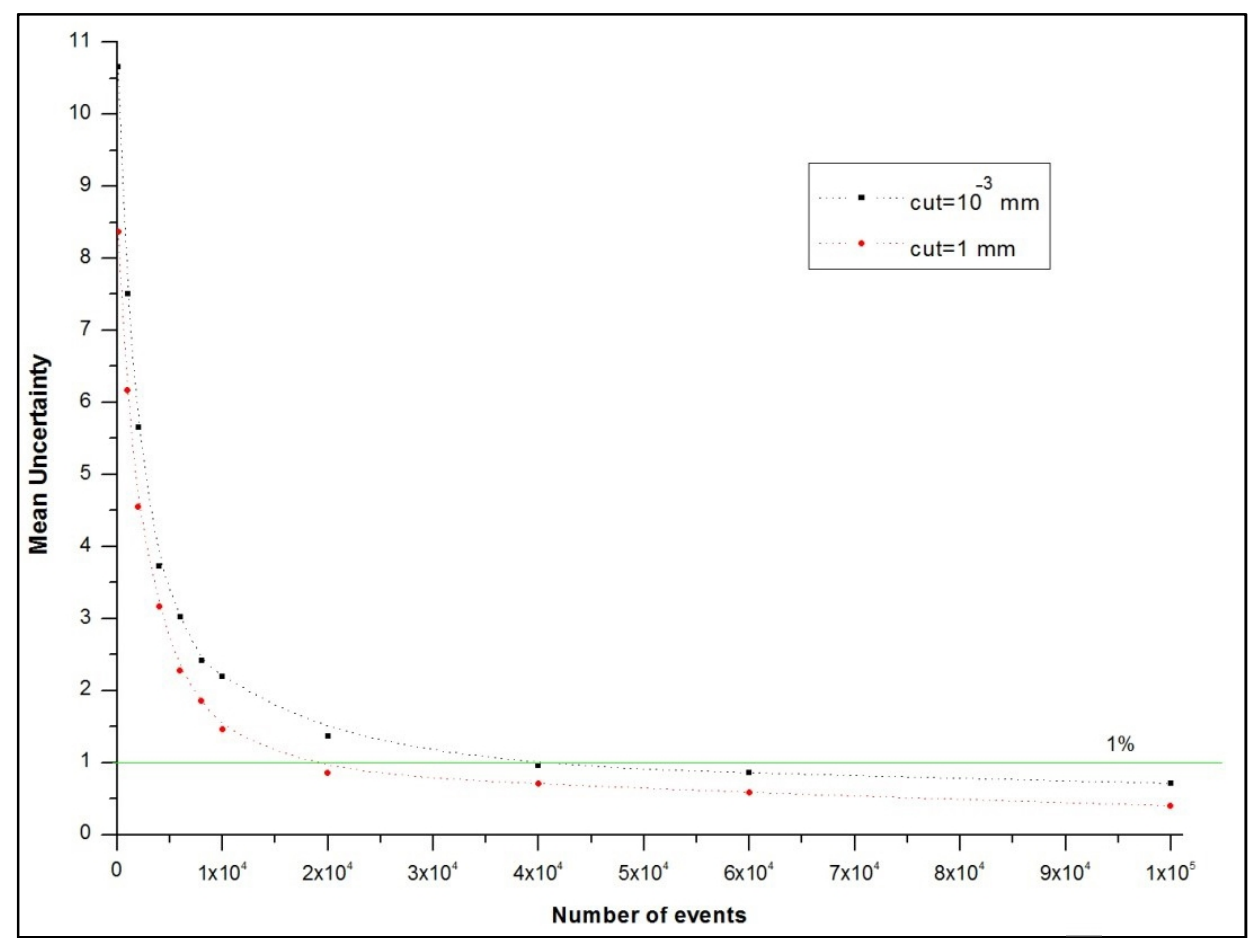

FIG. 4: The statistical uncertainty (\%) calculated for different values of number of events and for two setcut values (cut $=10^{-3} \mathrm{~mm}$, cut $=1 \mathrm{~mm}$ ).

\section{Statistical error on dose distribution calculation}

Method used to calculate the influence of Geant4 parameters on statistical uncertainty in each point of depth, is by the comparison of ten simulations according to equation 1 . In each simulation we change the value of random function in macro file by the command (/random/setseeds). The calcula- tion of statistical uncertainty $\sigma_{i}$ in point (i) of depth is carried out according to the following equation:

$$
\sigma_{i}=\frac{\left[D_{i 1}, D_{i 2}, \ldots, D_{i 10}\right]-\min \left[D_{i 1}, D_{i 2}, \ldots, D_{i 10}\right]}{2}
$$

where, $\left(D_{i 1}, D_{i 2}, \ldots, D_{i 10}\right)$ represent the energy deposit in slab" $i$ "on dose distribution for each simulation of 10 runs. 
The global number of slab (i) is 400 because the phantom was divided into $0.1 \mathrm{~mm}$ in $\mathrm{z}$ direction. The mean statistical error is calculated as the mean of $\sigma_{i}$.

\section{Results and Discussion}

The simulation represented in was obtained using the most accurate values $\left(10^{-3} \mathrm{~mm}\right.$ in cut, $10^{-2} \mathrm{~mm}$ step limit and $10^{5}$ events) presents a good agreement with the experimental data. Improving the validity of the model used. The studied parameters of Geant 4 are the number of events, the cut, the step limit, and the voxel size. These values of events, cut, and step limit are considered as reference for the next simulations. The Figure 4 shows the evolution of the statistical uncertainty calculated for different numbers of events and two values of set cut. The first (cut $=10^{-3} \mathrm{~mm}$ ) represents the lowest value of cut, and second is the highest value (cut $=1$ $\mathrm{mm}$ ). The $4 \times 10^{4}$ value corresponds to the lowest number of events which allows less than $1 \%$ of the mean statistical uncertainty for cut $=1 \mathrm{~mm}$ as recommended in beam delivery and properties report 78 of the international standards for radiation units (ICRU) ${ }^{8}$. This value of the number of events was selected as the minimum number needed to reach $1 \%$ of error with less accurate value of cut and it is used in the simulation to obtain the next results. The Figure 5 illustrates the evolution of the statistical uncertainty calculated for different values of set cut. The statistical uncertainty value increases with setcut value.

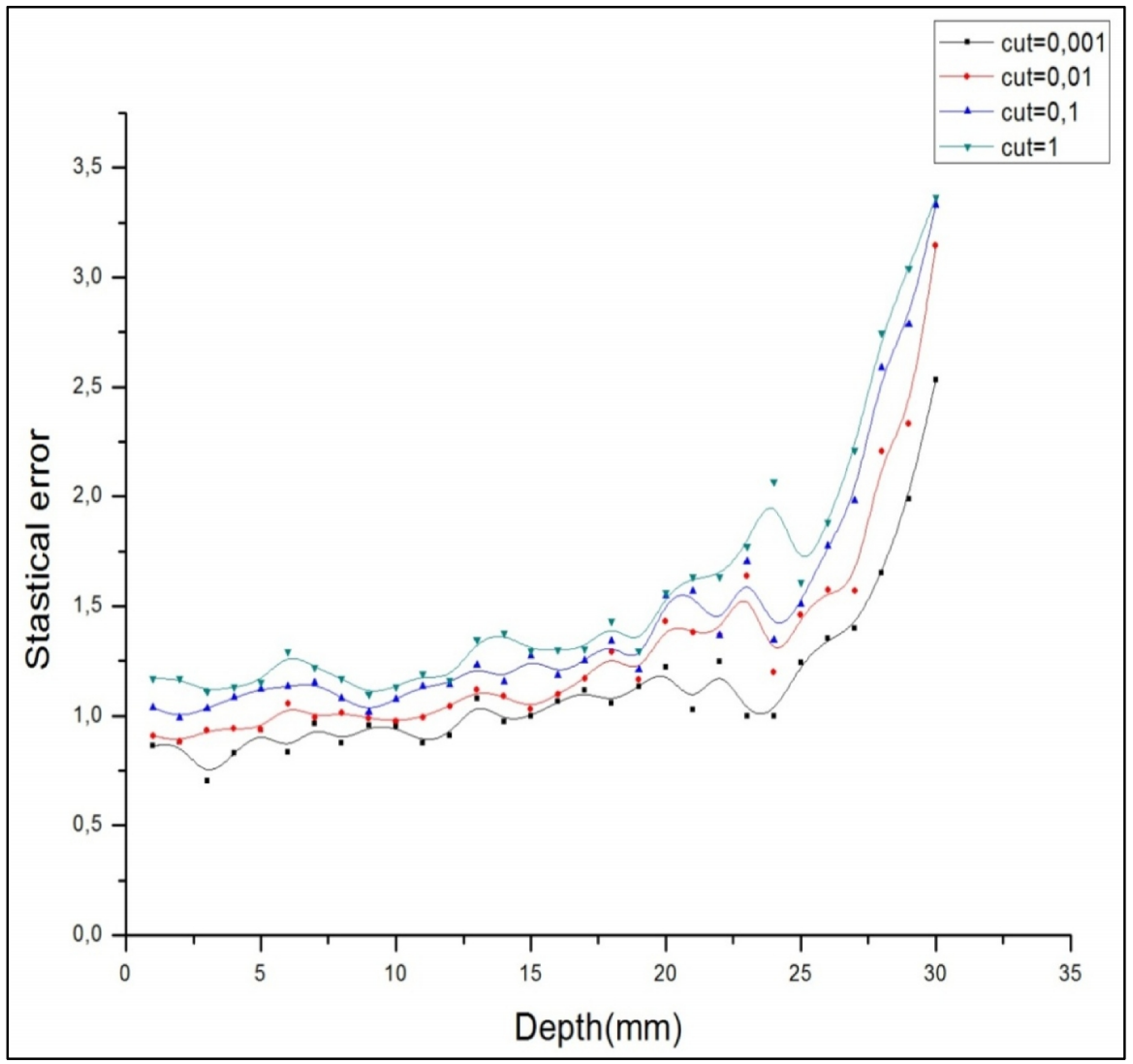

FIG. 5: The statistical uncertainty (\%) calculated for different values of setcut.

\section{Conclusion}

The obtained results show that the statistical uncertainty changes with the Geant 4 parameters like setcut and step limit. The cut and step limit values can be chosen in combination with the number of events to reach $1 \%$ precision recommended from ICRU in Monte Carlo codes. Using $4 \times$ $10^{4}$ events, $1 \mathrm{~mm}$ setcut and $10^{-2} \mathrm{~mm}$ step limit leads to an accurate simulation with a statistical uncertainty less or equal to $1 \%$.

\section{Conflict of interest}

The authors declare that they have no conflicts of interest. The authors alone are responsible for the content and writing of the paper.

\section{Acknowledgement}

This work was partially developed within the PCI2011 A1/035250/11 cooperation project between Universidad de Valencia and Mohammed V-Agdal University. 


\section{References}

1. Agostinelli S, Allison J, Amako K, et al. Geant4 - A simulation toolkit. Nucl Instrum Methods Phys Res A. 2003; A506:250-303.

2. Pia MG. The Geant4 Toolkit: simulation capabilities and application results. Nucl Phys B Proc Suppl. 2003;125:60-8.

3. GEANT4 Physics Reference Manual. Available from:

http://geant4.web.cern.ch/geant4/G4UsersDocume nts/UsersGuides/PhysicsReferenceManual/html/Ph ysicsReferenceManual.html. [Accessed date: 19/01/2015].

4. Zahra N, Frisson T, Grevillot L, et al. Influence of Geant4 parameters on dose distribution and computation time for carbon ion therapy simulation. Phys Med. 2010;26:202-8.

5. Gao J, Zheng Y. Monte Carlo study of secondary electron production from gold nanoparticle in proton beam irradiation. Int J Cancer Ther Oncol 2014; 2:02025.

6. Rana S, Pokharel S, Zheng Y, et al. Treatment planning study comparing proton therapy, RapidArc and intensity modulated radiation therapy for a synchronous bilateral lung cancer case. Int J Cancer Ther Oncol 2014; 2:020216.

7. Tesfamicael BY, Avery S, Gueye P, et al. Scintillating fiber based in-vivo dose monitoring system to the rectum in proton therapy of prostate cancer: A Geant4 Monte Carlo simulation. Int J Cancer Ther Oncol 2014; 2:02024.

8. Beam delivery and properties. Report 78. Journal of the ICRU. 2007;7:29-48. 